\title{
Sputter Ejection of Matter From Io
}

\author{
P. K. HAFF AND C. C. Watson ${ }^{1}$ \\ A. W. Wright Nuclear Structure Laboratory, Yale University, New Haven, Connecticut 06520
}

YUK L. YUNG

Division of Geological and Planetary Sciences, California Institute of Technology, Pasadena, California 91125

\begin{abstract}
The direct collisional interaction of magnetospheric particles with Io will lead to sputtering of atoms and molecules from the satellite into circum-Jovian space. The $\sim 520-\mathrm{eV} \mathrm{S}$ (and $\sim 260-\mathrm{eV} \mathrm{O}$ ) ions composing the Io torus are the most effective agents for net sputter removal of matter from the satellite. An incident flux of $\sim 10^{10} \mathrm{~cm}^{-2} \mathrm{~s}^{-1}$ is estimated to provide $\sim 5 \times 10^{10} \mathrm{~S}$ atoms $\mathrm{cm}^{-2} \mathrm{~s}^{-1}$ from sputtering of a (dayside) atmosphere with an exobase at a few hundred kilometers and up to $\sim 10^{12} \mathrm{~S}$ atoms cm $\mathrm{cm}^{-2} \mathrm{~s}^{-1}$ from an atmosphere at $1500^{\circ} \mathrm{K}$ with an exobase at $\sim 2.2 R_{\mathrm{I}}$. The supply of $\mathrm{S}$ (and $\mathrm{O}$ ) required to stabilize the torus has been estimated by others to be from $10^{10}$ to $10^{12} \mathrm{~cm}^{-2} \mathrm{~s}^{-1}$. If $\mathrm{Na}$ and $\mathrm{K}$ are present in the atmosphere at a concentration level of $1 \%$, the corresponding sputtering rates are calculated to be a few times $10^{8} \mathrm{~cm}^{-2} \mathrm{~s}^{-1}$ for an exobase at several hundred kilometers. These numbers are large enough to supply the $10^{7} \mathrm{~cm}^{-2} \mathrm{~s}^{-1}$ fluxes required to maintain the $\mathrm{Na}$ and $\mathrm{K}$ clouds. Sputtering can also remove heavy molecules, like $S_{n}$, from the atmosphere. At night, direct $S$ sputtering of the unprotected surface is calculated to eject $S$ and $\mathrm{Na}$ (1\% concentration) at rates given approximately by $\sim 10^{10}$ and $\sim 10^{8} \mathrm{~cm}^{-2} \mathrm{~s}^{-1}$, respectively. All atomic species residing on the surface must be ejected into circum-Jovian space at a rate approximately proportional to their (surface) abundances, if direct surface sputtering occurs, so that the particle content of the inner Jovian magnetosphere should map rather faithfully all species present on Io's surface. The processes of plume sputtering, avalanche cascading, and ionic saltation may lead to spatial and temporal variations in the number of ejected particles.
\end{abstract}

\section{INTRODUCTION}

The discovery by Brown [1974] of a sodium cloud associated with the orbit of the innermost Galilean satellite, Io, and the subsequent identification of a potassium cloud [Trafton, 1975a] and a torus of ionized sulfur [Kupo et al., 1976] and oxygen [Pilcher and Morgan, 1979] have led to a consideration of mechanisms which would be energetic enough to eject the observed species from Io into circum-Jovian space. In order for the clouds to be maintained in a steady state condition, source strengths of $\sim 10^{7} \mathrm{Na}$ atoms $\mathrm{cm}^{-2} \mathrm{~s}^{-1}$ [Brown and Yung, 1976] and from $10^{10}$ to $10^{12} \mathrm{~S}$ (and $\mathrm{O}$ ) atoms $\mathrm{cm}^{-2} \mathrm{~s}^{-1}$ [Kumar, 1979] are required. (All sputter fluxes will be referenced to Io's surface.) Jeans escape is too inefficient to account for these emission rates at likely atmospheric temperatures, which might be in excess of $\sim 1000^{\circ} \mathrm{K}$ [Brown and Yung, 1976]. Observations of the $\mathrm{Na}$ cloud, moreover, imply that the $\mathrm{Na}$ atoms are ejected with velocities of the order of several kilometers per second, well in excess of thermal velocities [Carlson et al., 1978; Trafton, 1975b]. Sputtering is a suitably energetic process and one which is expected to occur on Io [Carlson et al., 1978; Matson et al., 1974]. Particles ejected from a medium via sputtering typically have energies of the order of several electron volts, which is of the order of magnitude observed for $\mathrm{Na}$ ejected from Io. Sputtering is also capable of ejecting both neutral and ionized particles and so could be directly responsible for the neutral $\mathrm{Na}$ and $\mathrm{K}$ clouds.

We consider here the possible efficiencies of sputtering processes for several models of the interaction of the particle flux with Io. Sputtering occurs when fast ions transfer a por-

\footnotetext{
${ }^{1}$ Now at W. K. Kellogg Radiation Laboratory, California Institute of Technology, Pasadena, California 91125.
}

Copyright (C) 1981 by the American Geophysical Union. tion of their energy to 'target' atoms, some of which as a consequence can escape from the target surface. On Io the dominant flux species for sputtering will be composed of corotating $\mathrm{S}(\sim 520 \mathrm{eV})$ and $\mathrm{O}(\sim 260 \mathrm{eV})$ ions. $S$ ions are somewhat more efficient at sputtering, and for simplicity we consider their effects alone; such an approximation is within the expected accuracy of the calculations to be discussed. These ions are trapped in the magnetosphere in the vicinity of Io's orbit, and the corotation velocity of the Jovian magnetic field $(\sim 56 \mathrm{~km} /$ s) corresponds to the particle energies given above. The flux of $S$ ions incident on Io is taken to be $\sim 10^{10} \mathrm{~cm}^{-2} \mathrm{~s}^{-1}$. $S$ ions of this energy are expected to have sputtering yields (number of target particles out per number of $S$ ions in) of the order of unity [Sigmund, 1969] for many solid targets. This yield would be barely sufficient to provide the required number of $S$ atoms, although it is possibly adequate to account for ejection of alkali atoms. However, the low cohesive energy of $\mathrm{SO}_{2}$ frost, a likely surface constituent on Io [Smythe et al., 1979; Fanale et al., 1979], suggests that sputtering yields for this material under low-energy ion bombardment may be up to an order of magnitude greater than those for pure $S$ or for silicates. We will consider sputtering yields for both classes of targets at a later point in this paper.

\section{Mass Loss by ATMOSPHERIC SPUTTERING}

Direct sputter removal of particles from the surface is possible only if the atmosphere of Io is sufficiently thin. The Voyager IRIS experiment provides evidence for the presence of a gaseous $\mathrm{SO}_{2}$ phase, and the implied pressure of $\sim 10^{-7}$ bar is comparable with the vapor pressure of solid $\mathrm{SO}_{2}$ at a (dayside) surface temperature of $130^{\circ} \mathrm{K}$ [Pearl et al., 1979]. A pressure much in excess of $\sim 10^{-11}$ bar would be great enough to prevent most sputtered atoms from leaving the satellite directly, and a pressure only slightly greater would suffice to shield the surface altogether from the effects of incident $S$ ions. At the 
lower nighttime temperatures $\left(\leq 110^{\circ} \mathrm{K}\right)$ we may expect the atmospheric pressure to drop dramatically as the $\mathrm{SO}_{2}$ condenses. Consequently, the atmosphere is likely to be inhomogeneous on a global scale; on the dayside the surface may be at least partially protected from charged particle bombardment, while on the nightside, direct ejection of particles from the surface may be possible. We are thus led to consider sputter ejection of particles both from the solid surface and from the atmosphere.

We will consider several probable scenarios in what follows. In each case the estimated sputter-induced mass loss rate may well be applicable to only a limited region of Io's surface. An estimate of the global average source flux necessarily entails specific assumptions concerning the composition and structure of the atmosphere and/or surface, as well as the distribution of the impinging ion flux, none of which are well known. However, given such assumptions, the present estimates may readily be appropriately scaled by the reader. The sputtered loss flux should vary approximately linearly with the incident ion flux, for instance. Moreover, the estimates presented here, taken as they stand, represent limiting values for the loss rates, and it is in this context that they will be discussed.

When the kinetic energy of the impinging ion is sufficiently low that it interacts with target atoms primarily through screened Coulomb collisions, the resulting collisional cascades, and thus the sputtering yields, are well described by classical kinetic transport theory. Significantly, the yield calculated in this formalism is independent of the target's density, to a good approximation. Accurate yield expressions for the case of a solid surface have been given by Sigmund [1969]. In atmospheric sputtering [Haff et al., 1978; Haff and Watson, 1979; Watson et al., 1980a, b] the gravitationally bound gas functions as the target, and atoms or molecules can be sputtered from the exobase region if they are given sufficient energy through recoil collisions to escape the gravitational field. Our estimates for the atmospheric case are based on the analysis developed by Haff and Watson [1979], applied for illustrative purposes to a $100 \% \mathrm{SO}_{2}$ atmosphere. These authors show that the atmospheric sputtering yield is inversely proportional to $U$, the gravitational binding energy of the molecule at the exobase. We find that with $U=2.2 \mathrm{eV}$ for escape (to infinity) from $\sim 1 R_{\mathrm{lo}}$, the sputtering yield for $\mathrm{SO}_{2}$ molecules is $Y_{\mathrm{SO}_{2}} \sim$ 5. Accompanying these molecules will be a smaller number of $\mathrm{S}$ and $\mathrm{O}$ atoms arising from the breakup of the $\mathrm{SO}_{2}$ molecule in the initial collision. The liberated molecules will subsequently be dissociated, and the $S$ and $O$ atoms ionized by the ambient magnetospheric electron flux. This leads to a source current of $\sim 5 \times 10^{10} \mathrm{~S}$ ions $\mathrm{cm}^{-2} \mathrm{~s}^{-1}$, which may be sufficient to stabilize the $S$ torus. (We comment on the $O$ source strength below.) Actually, this estimate of the escape rate from $\sim 1 R_{\mathrm{Io}}$ is conservative. The presence of Jupiter's gravitational field allows particle escape from Io to occur at approximately $5.3 R_{\mathrm{Io}}$ [McElroy and Yung, 1975]. The energy required for escape from near $1 R_{\mathrm{lo}}$ is approximately $70 \%$ of the amount which would be necessary in the absence of Jupiter's field. The effective escape flux is thus increased by about $40 \%$, that is, to $\sim 7 \times 10^{10} \mathrm{~cm}^{-2} \mathrm{~s}^{-1}$.

On small gravitating bodies there is a property of hot atmospheres which may substantially increase the emission rate. If, neglecting the effect of the proximity of Jupiter for the moment, we take account of the variation of the gravitational acceleration with radius $r$, the (isothermal) density distribution has the form $n(r) \propto \exp (G m M / k T r)$, where $m$ is the mass of the $\mathrm{SO}_{2}$ molecule, $M$ is the satellite mass, and $G$ is the gravitational constant. This formula is good for calculating $n(r)$ up to the exobase. Above that level, $n(r)$ is determined by the Keplerian orbits of particles [Chamberlain, 1963]. We consider a few typical $\mathrm{SO}_{2}$ model atmospheres. Case $\mathrm{A}$ corresponds to an isothermal atmosphere at $1000^{\circ} \mathrm{K}$ with $n(z=100 \mathrm{~km})=1 \times$ $10^{10} \mathrm{~cm}^{-3}$, where $z$ is the altitude above the satellite surface. Cases $B$ and $C$ assume an isothermal atmosphere with $T=$ $1500^{\circ} \mathrm{K}$. In case $\mathrm{B}, n(z=100 \mathrm{~km})=1 \times 10^{9} \mathrm{~cm}^{-3}$, and in case C, $n(z=100 \mathrm{~km})=1 \times 10^{10} \mathrm{~cm}^{-3}$. All three cases satisfy the constraint that the disk-averaged column abundance of $\mathrm{SO}_{2}$ is less than the UV spectroscopic limit of $0.005 \mathrm{~cm}$ atm. The exobase, from which sputtering occurs, can potentially attain a height of up to $\sim 2.2 R_{\mathrm{Io}}$ (case C), with a corresponding decrease in the escape energy to $U \sim 1.0 \mathrm{eV}$ (for $\mathrm{SO}_{2}$ ). Since the sputtering yield is inversely proportional to $U$, the effective emission current of $\mathbf{S}$ in this model, referred to the satellite surface, would be $\sim 1.0 \times 10^{12} \mathrm{~S} \mathrm{~cm}^{-2} \mathrm{~s}^{-1}$, of the order of the maximum yield rate required for torus stabilization. The effect of the Jovian gravitational field is more pronounced when the exobase is at high altitude. At $2.2 \boldsymbol{R}_{\mathrm{lo}}$ the escape energy of $1.0 \mathrm{eV}$ for $\mathrm{SO}_{2}$ used in the above calculations becomes $\sim 0.58$ $\mathrm{eV}$ [McElroy and Yung, 1975], and the effective emission current, referred to Io's surface, increases from $\sim 1.0 \times 10^{12}$ to $\sim 1.7 \times 10^{12} \mathrm{~cm}^{-2} \mathrm{~s}^{-1}$. The Jeans escape fluxes for $\mathrm{SO}_{2}$ molecules for cases $A, B$, and $C$ above (with the exobase at 1.6, 1.7, and $2.2 R_{\mathrm{Io}}$, respectively) are $3.2 \times 10^{7}, 1.2 \times 10^{9}$, and $1.5 \times$ $10^{10} \mathrm{~cm}^{-2} \mathrm{~s}^{-1}$, respectively. These flux values include the effect of the presence of Jupiter.

We note that a reduction of these full-disk values by a factor even slightly smaller than unity would probably be sufficient to eliminate the thermal loss models. To transform the results given here to an atmosphere of more restricted areal coverage is a matter of multiplication only.

Other ionic species will also be ejected from the atmosphere by sputtering, roughly in proportion to their abundance. Ignoring the effects of diffusive separation, which will lead to enhanced sputter emission of lighter molecules [Watson et al., $1980 a, b]$, we find that if $\mathrm{Na}$ and $\mathrm{K}$ are present in the form of the sulfides $\mathrm{Na}_{2} \mathrm{~S}$ and $\mathrm{K}_{2} \mathrm{~S}$, then $Y_{\mathrm{Na}_{2} \mathrm{~S}} \sim 0.04$, and $\mathrm{Y}_{\mathrm{K}_{2} \mathrm{~S}} \sim 0.03$ (for $1 \%$ abundance of both molecular species, the remaining atmosphere being composed of $\mathrm{SO}_{2}$ ). These values assume an exobase in the vicinity of the satellite surface, and the effective emission fluxes are $\sim 4 \times 10^{8} \mathrm{Na} \mathrm{cm}^{-2} \mathrm{~s}^{-1}$ and $\sim 3 \times 10^{8} \mathrm{~K}$ $\mathrm{cm}^{-2} \mathrm{~s}^{-1}$. For an exobase at $\sim 2.2 R_{\mathrm{Io}}$ these fluxes, referred to the planetary surface, increase by about a factor of 10 . These values are significantly larger than the $\sim 10^{7} \mathrm{~cm}^{-2} \mathrm{~s}^{-1}$ flux required to maintain the $\mathrm{Na}$ and $\mathrm{K}$ clouds. However, the atmospheric concentration of these elements may be lower than the $1 \%$ values used here.

\section{MASS LosS by SPUTTERING of Io's SuRface}

\section{Sputtering of Volatile and Insulating Targets}

Even if no $\mathrm{Na}$ or $\mathrm{K}$ compounds were contained in the atmospheric inventory, sputter ejection of these species, as well as $\mathrm{S}$, might occur from the unprotected nightside of Io. The calculation of the total number of atoms sputtered in this case requires caution. Brown et al. [1978] have shown that water ice sputters, under energetic $\mathrm{H}, \mathrm{He}$, and $\mathrm{O}$ bombardment, at a rate which is several orders of magnitude larger than that predicted from the standard collision cascade theory [Sigmund, 1969]. If $\mathrm{H}_{2} \mathrm{O}$ sputters in this way, other volatiles, such as $\mathrm{SO}_{2}$ frost, might behave in a similar fashion. Furthermore, anoma- 
lous sputtering yields have been reported for ion bombardment of some alkali salts [Biersack and Santner, 1976].

Unfortunately, there is no tested theory of these sputtering processes, although a model of enhanced sputtering due to Seiberling et al. [1980a, b], when applied to experimental results on the special material $\mathrm{UF}_{4}$, seems to give a semiquantitative and consistent description of the ion erosion process. This work and earlier speculations [Haff, 1976], as well as recent data on ice obtained by Brown et al. [1980], all indicate that the energy dependence of the yield $Y$ is a function of the electronic stopping power $d E / d x$ ), rather than the nuclear stopping power $d E / d x)_{n}$. It is the nuclear stopping power which mediates the usual collisional sputtering yield [Sigmund, 1969]. In the case of 500-eV S ion bombardment of the exposed surface of Io we argue that the sputtering process ought to be principally collisional, since the nuclear stopping is large and the projectiles are poorly ionizing. This observation is supported by the investigations of Griffith et al. [1980], who discovered that the anomalous sputtering yield of $\mathrm{UF}_{4}$ for highly ionizing beams gave way to the expected collisional yield at lower energies, where nuclear stopping is important.

Since the sputtering yield in the collisional regime is inversely proportional to $W$, the energy required to remove a particle from the target, we need to distinguish between the sputtering of condensed $\mathrm{SO}_{2}(W \sim 0.37 \mathrm{eV})$ and the sputtering of more strongly bonded substances (e.g., for $\mathrm{S}, W \sim 2.9 \mathrm{eV}$ ). Furthermore, in the case of a weak molecular solid like $\mathrm{SO}_{2}$ there may be competition between the emission of molecules and that of individual atoms. No general theory has been advanced to describe low-energy sputtering of such substances, and we can discuss here only simplified limiting cases. Thus to be definite, we choose $W$ to have the typical value $4 \mathrm{eV}$ for all target species. (This will lead to a conservative estimate of the flux for a pure $\mathbf{S}$ target.)

We find that the sputtering yields for $\mathrm{S}, \mathrm{Na}$, and $\mathrm{K}$ are $Y_{\mathrm{S}} \sim$ 0.89 and $Y_{N_{a}} \sim Y_{K} \sim 0.009$ for a surface composed principally of S-containing compounds with an admixture of $\sim 1 \%$ each of $\mathrm{Na}$ and $\mathrm{K}$ atoms. The corresponding emission rates are $\sim 9 \times$ $10^{9} \mathrm{~S}$ atoms cm $\mathrm{cm}^{-2} \mathrm{~s}^{-1}$ and $\sim 9 \times 10^{7} \mathrm{Na}$ and $\mathrm{K}$ atoms cm $\mathrm{cm}^{-2} \mathrm{~s}^{-1}$. Evidently, direct $S$ emission from the surface can only resupply the flux swept up by Io (taken to be $10^{10} \mathrm{~cm}^{-2} \mathrm{~s}^{-1}$ ); for example, diffusive losses would need to be supplied by another mechanism. The alkali atom flux, however, seems to be large enough to maintain the $\mathrm{Na}$ and $\mathrm{K}$ clouds.

\section{Sputtering of Adsorbed Molecules}

For the case of an $\mathrm{SO}_{2}$ molecule adsorbed on a lighter substrate like $\mathbf{S}$, no sputtering model has been developed. If we leave aside the question of how the cross sections enter into such a calculation, the binding energy dependence of the atomic yield, given by the expression $(W+2 U) /(W+U)^{2}$, would imply an increase in the $\mathrm{SO}_{2}$ flux $\left(W_{\mathrm{SO}_{2}} \sim 0.37 \mathrm{eV}\right)$ over an escape flux of atomic $S$ from a pure $S$ target by about a factor of 3. This translates into a total atomic escape flux of $\sim 3 \times$ $10^{10}$ atoms cm $\mathrm{cm}^{-2} \mathrm{~s}^{-1}$, which would be a modest source of particles for the torus. This calculation assumes that $\mathrm{SO}_{2}$ molecules may be removed intact from Io by collisional momentum transfer with surrounding atoms and molecules. A $500-\mathrm{eV}$ incident $\mathrm{S}$ ion has enough energy to dissociate many $\mathrm{SO}_{2}$ molecules. However, most of the sputtered particle flux originates from low-energy collisions between daughter recoils. These collisions are presumably gentle enough to remove many $\mathrm{SO}_{2}$ molecules without dissociation.

\section{Effect of Surface Topography}

Another feature which we will mention only briefly has to do with surface topography on a small scale. Regardless of details of the surface composition we can assert that surface roughness characteristics will have an effect on the total sputtering yields. All estimated sputtering rates quoted above assume a smooth surface. However, surface roughness can occur due to micrometeorite gardening of the outermost planetary layer, due to chemical and thermal effects, or due to the effects of sputtering itself. On Io the fast volcanic resurfacing rates (0.1-1.0 mm $\mathrm{yr}^{-1}$ [Johnson et al., 1979], compared to sputter processing rates $\left(10^{-4}-10^{-5} \mathrm{~mm} \mathrm{yr}^{-1}\right)$, indicate that surface properties are controlled to a large extent by volcanic activity. Matson et al. [1981] suggest, on the basis of the thermal properties of Io, that its surface may be porous and fluffy. Such a surface would probably sputter at a rate which is slower than that expected for a smooth surface of the same composition, because particles sputtered from one location may be caught by a nearby protrusion, therefore never really leaving the surface. Estimates of the magnitude of the reduction in the sputtering yield due to this effect range from a factor of $\sim 2$ to factors as large as $\sim 10$ [Carey and McDonnell, 1976; Hapke and Cassidy, 1978]. Although this effect would make it more difficult to erode sufficient $\mathbf{S}$ from the surface to supply the torus than our earlier estimates indicated, it may still be possible to eject enough $\mathrm{Na}$ and $\mathrm{K}$ atoms.

\section{Energy Spectra of Sputtered Particles}

We turn now briefly to a consideration of the energy spectrum of emitted particles. Molecules sputtered from the atmosphere will have a spectrum of the form

$$
\phi_{A}(\varepsilon) d \varepsilon \propto d \varepsilon / \varepsilon^{2}
$$

where $\varepsilon$ is the energy of the sputtered particle at the exobase. On the other hand, if particles are emitted from the solid planetary surface, the spectrum at the moment of emission is of the form

$$
\phi_{\mathrm{SOL}}(\varepsilon) d \varepsilon \propto \varepsilon d \varepsilon /(\varepsilon+W)^{3}
$$

where $W$ is the chemical binding energy at the surface. At the limbs of the satellite a third, related, emission process can occur. Collision between atmospheric gas molecules and magnetospheric ions can lead to direct emission of the struck molecules (or, more likely, one of their atomic constituents) [Carlson et al., 1978; Watson et al., 1980a, b]. This one-step process produces higher-energy recoils than the typical multistep sputtering process. The spectrum has the approximate form

$$
\phi_{D}(\varepsilon) d \varepsilon=\sigma_{0} d \varepsilon / E
$$

where $E$ is the energy of the incident ion, and $\sigma_{0}$ is a constant. It can be shown (C. C. Watson and P. K. Haff, unpublished calculations, 1980) that the average number of such high-energy atmospheric atoms emitted per incident ion is generally less than 0.5. Therefore the maximum associated atomic escape flux would be $\sim 5 \times 10^{9} \mathrm{~cm}^{-2} \mathrm{~s}^{-1}$, and the actual value is probably very much smaller.

Presumably, there is a smooth gradation of this direct knock-on process into the more usual cascade picture as the initial collision altitude decreases. The higher-energy particles, and in the case of partially developed cascades their immediate daughters, will tend to be directed preferentially in 
the direction of the plasma motion, so that the fastest particles sputtered from the atmosphere would lead the satellite orbit. This direct ejection mechanism has been studied for solar wind impact on Mars and Venus [Watson et al., 1980a, b], but analogous detailed calculations have not yet been performed for $\mathbf{S}$ impact on Io. However, the above estimates suggest that direct ejection will contribute significantly only to the highenergy portion of the escape spectrum, which contains relatively few particles. We will therefore not discuss this mechanism more fully at the present time, except to note that other atomic species will be ejected essentially in proportion to their abundance at the altitudes where the initial collisions occur.

It is important to emphasize that while the expression for $\phi_{D}$ refers mainly to sputtered atoms, the expression $\phi_{A}$, and in some cases $\phi_{\text {sol }}$, refers to the molecular sputtering spectrum. If $\mathrm{Na}$, for example, were removed primarily in molecular form from the atmosphere, then the observed velocity distribution of neutral $\mathrm{Na}$ atoms cannot be obtained by the use of $\phi_{A}$ alone. The effects of molecular dissociation must be added by an appropriate summation of particle velocities in order to obtain the final energy or velocity distribution.

\section{Sputtering by Oxygen Ions and the Oxygen Ejection Flux}

The presence of an incident $O$ flux will lead to sputtering of the surface and atmosphere in much the same way as does the $S$ flux. At the present level of our calculational ability, and with the existing uncertainties in the flux values and in the 'target' structure, it is not yet worthwhile to consider $O$ effects separately. For purposes of comparison we note that the estimated molecular yield from an $\mathrm{SO}_{2}$ atmosphere (exobase at $\sim 1 R_{\mathrm{I}}$ ) for 260 -eV O ions is $Y_{\mathrm{SO}_{2}} \sim 3$. Limits on the observed densities of these two ions in the torus will provide constraints on the sputtering process.

Although we might expect the $O$ emission rate to be about twice that of S, deviations from that ratio could arise, for example, from diffusive separation and preferential ejection of lighter atmospheric constituents. For the moment we can only say that rates for ejection of $O$ ought to be comparable in magnitude to $S$ ejection rates, but factors of the order of unity cannot yet be calculated reliably.

\section{Sputtering by the Proton Flux}

The proton flux at Io can also cause sputtering. This was the original mechanism suggested by Matson et al. [1974] for $\mathrm{Na}$ emission. For a collisional sputtering mechanism, protons with energies centered, quite broadly, at $\sim 1 \mathrm{keV}$ will have the largest sputtering yields. The actual yield values for proton bombardment in this energy range will be up to several orders of magnitude smaller than those due to heavy ions like $O$ and $S$, because of mass mismatch and smaller interaction cross sections characterizing the proton scattering in the target. For proton bombardment of a pure $S$ surface, for instance, collisional yields of the order of $Y_{S} \sim 0.04$ (for emission from the satellite) might be expected. For a $1 \% \mathrm{Na}$ admixture a low-energy proton flux of $\sim 10^{11} \mathrm{~cm}^{-2} \mathrm{~s}^{-1}$ would be required to eject $\sim 4 \times 10^{7} \mathrm{Na}$ atoms $\mathrm{cm}^{-2} \mathrm{~s}^{-1}$. The corresponding $\mathrm{S}$ emission flux would be $\sim 4 \times 10^{9} \mathrm{~cm}^{-2} \mathrm{~s}^{-1}$. Enhanced sputtering processes might occur for Io's surface materials under proton bombardment, as in the case for $\mathrm{H}_{2} \mathrm{O}$ ice, but the energy spectrum of emitted particles is probably too soft to enable the particles to escape from Io. This last statement is based on expectations suggested by the model of Seiberling et al. [1980a, b], but at present, experimental confirmation is lacking.

Proton sputtering of the $\mathrm{SO}_{2}$ atmosphere would likely be a more important source of heavy ion emission from Io. Estimated atmospheric $\mathrm{SO}_{2}$ yields for 1-keV proton impact are of the order of $Y_{\mathrm{SO}_{2}} \sim 0.07$, so even a flux as large as $10^{11}$ protons $\mathrm{cm}^{-2} \mathrm{~s}^{-1}$ would give only $\sim 7 \times 10^{9}$ molecules $\mathrm{cm}^{-2} \mathrm{~s}^{-1}$, still smaller than likely emission rates.

\section{Ionic Saltation and Related Processes}

Matson et al. [1974] pointed out that if sputtered atoms become ionized, then existing electric and magnetic fields could act to accelerate these ions back into the surface. Potential differences as large as $600 \mathrm{kV}$ between Io's surface and the surrounding plasma could cause heavy ion impact in an energy regime where sputtering is very efficient $(Y \sim 1-10)$ [Sigmund, 1969]. To use a rather arbitrary example, if we consider $S$ bombardment having an intrinsic yield $Y_{\mathrm{S}}$ and assume that a fraction $p$ of the sputtered atoms were subsequently ionized and reimplanted at the same energy, then the resulting yield (of neutral atoms) for a single initial incident particle would be

$$
Y_{\mathrm{S}}^{\text {TOT }}=Y_{\mathrm{S}}(1-p)\left(1-p Y_{\mathrm{S}}\right)^{-1}
$$

If $Y_{\mathrm{s}}>1$, then $Y_{\mathrm{s}}^{\text {TOT }}>Y_{\mathrm{S}}$, and the mechanism leads to an enhancement of the yield. Note, however, that this equation loses meaning when $p Y_{s} \geq 1$. In this case the ion 'cascade' becomes self-sustaining and could in principle continue even in the absence of the initiating magnetospheric flux. The avalanche would increase in intensity with time until space charge or other effects acted to limit its growth. It therefore seems that, at least locally, very intense sputter erosion could occur. The distribution of field-induced sputtering over the surface of Io and the variation in time of such activity would depend upon the changing electromagnetic field configuration. As was pointed out by Matson et al. [1974], such cascade effects may be responsible for temporal changes in the column density of $\mathrm{Na}$ which have been observed to occur over periods of a few hours.

It is not necessary for the ionization processes to occur after the atom has been ejected in order for enhanced sputtering effects to occur. In some substances a fraction of the sputtered flux originates in the form of ions [Benninghoven, 1973; Griffith et al., 1980], although this is not a universal phenomenon. In configurations wherein the local electric field intersects the surface at a large angle with respect to the normal, such ions can be reimplanted at energies where sputtering yields are large. If the average yield of ions from such an impact exceeds unity, then a chain reaction occurs which propagates over the surface in the downfield direction. The runaway nature of the process will, again, ultimately be limited by charging effects. This transport mechanism, which resembles in many respects the transport of wind-blown sand, may be termed ionic saltation. The saltation process is ordinarily one of lateral movement of ions and to that extent has more to do with local surface erosion and redeposition than ejection of matter into space. However, neutral atoms will also be sputtered along with the ionic component, and the most energetic of these will be able to escape. Like the cascade effect, ionic saltation would also lead to time and space variation of emitted particle fluxes. 


\section{Sputtering of Elements Other Than $\mathrm{S}, \mathrm{O}, \mathrm{Na}$, and $\mathrm{K}$}

We return now to a discussion of the more conventional sputtering processes. So far we have discussed emission of a limited variety of atomic species, namely, $S, O, \mathrm{Na}$, and $\mathrm{K}$. However, sputtering by $S$ (and $O$ ) ions will lead to ejection into circum-Jovian space of any atomic species found on the extreme outer satellite surface or in an atmospheric molecule. If $n_{i}$ is the (small) fractional abundance of a species $i$ in the outer few monolayers of a sulfur particle resting on the surface of $\mathrm{Io}$, then the total sputtering yields, for example, of $\mathbf{M g}$, $\mathrm{Ca}, \mathrm{Zn}, \mathrm{Ag}, \mathrm{Pb}$, and $\mathrm{U}$ atoms can be estimated to be $Y_{\mathrm{Mg}} / n_{\mathrm{Mg}}$ $=0.91, Y_{\mathrm{Ca}} / n_{\mathrm{Ca}}=0.87, Y_{\mathrm{Zn}_{\mathrm{n}}} / n_{\mathrm{Zn}_{\mathrm{n}}}=0.82, Y_{\mathrm{Ag}} / n_{\mathrm{Ag}}=0.55$, and $Y_{\mathrm{U}} / n_{\mathrm{U}}=0.51$. These results assume a uniform value of $4 \mathrm{eV}$ for the chemical barrier energy. Variations in $W$ will affect the yields only slightly, however. Various ionic species have been identified in the Jovian magnetosphere [Sullivan and Bagenal, 1979]. Peaks in the mass to charge $\left(A / Z^{*}\right)$ spectrum at $A / Z^{*}$ $=104 \pm 5$ and $A / Z^{*}=160 \pm 10$ were attributed to molecular ions, but it would clearly be possible to produce the appropriate atomic species (say, Ag and a rare earth element) if they existed on an unprotected surface. In fact, if $\mathrm{Na}$ and $\mathrm{K}$ come from the surface via sputtering, then it is completely certain that any other element present there will also be introduced into the Jovian magnetosphere at a rate which is roughly proportional to its abundance. From this point of view the most pressing question involving the introduction of matter into the magnetosphere from Io is not whether sputtering can account for the few species which are observed, but rather, where is everything else? Although we may believe that $S$ and $O$ occupy a substantial fraction of the atomic sites in the extreme outer monolayer of the satellite, it strains belief that they, together with perhaps a couple of alkali species, occupy all of the sites. On a planetary body as active as Io, where the communication between the surface and the interior in terms of mass transport is much greater than elsewhere in the solar system, we might rather expect to see a better representation of the lighter abundant elements than on a geologically more quiescent body (like the earth). Most methods of removing matter from the surface run into this difficulty. The problem might be ameliorated to some extent by demanding the existence of a sufficiently thick global atmosphere that would prevent any direct surface sputtering, but one would then need to explain why alkali compounds (to supply the $\mathrm{Na}$ and $\mathrm{K}$ clouds), but not others (containing carbon, chlorine, fluorine, etc.), are present.

\section{Sputtering of Exotic Molecular Species}

Various exotic molecular species could also come from the sputtering of atmospheric molecules. Molecular ejection is the principal mass loss mode for atmospheric sputtering, and thus there potentially exists a suitable source of particles which could be subsequently ionized to form molecular ions with a high $A / Z^{*}$ ratio. These need not be restricted to species like $\mathrm{SO}_{2}{ }^{+}, \mathrm{S}_{2}{ }^{+}$, etc., but could be more complicated if the Ionian atmosphere contained the appropriate species. Thus complexes composed of a number $\boldsymbol{n}$ of $\mathrm{S}$ atoms, $\mathrm{S}_{n}$, can be removed from Io via sputtering with a yield given approximately by $Y_{\mathrm{s}_{n}} / n_{\mathrm{s}_{n}} \sim 2 Y_{\mathrm{SO}_{2}} / n$, where $n_{\mathrm{s}_{n}}$ is the (small) fractional abundance of $S_{n}$. This formula is approximately valid if $n$ is not too large. The emission of particles with very large $n$, that is, small dust particles, has been ascribed to electromagnetic forces by Johnson et al. [1980]. Sputtering will be ineffective at removing these particles (although it can play a role in their destruction), and we do not consider them further. In an isothermal, predominantly $\mathrm{SO}_{2}$, atmosphere in equilibrium, heavy molecules like $S_{n}$, where $n$ is larger than two, will be sputtered at a considerably slower rate than their bulk abundance $n_{\mathrm{s}_{\mathrm{n}}}$ might otherwise imply, because of the effects of diffusive separation. However, on Io the presence of energizing sources in the form of volcanoes may enhance the abundance of heavy molecules at high altitudes, so that sputtering of these species can occur. The volcanoes may evidently also serve as the source of the large molecules in question. Direct sputtering of an atmosphere containing some percentage of heavy molecules which is turbulently mixed at extreme altitudes, or which contains a large concentration of such species in bulk, may be one of the few mechanisms for injecting whole, complex, heavy molecules from the planet into space.

\section{Sputtering of Volcanic Plumes}

The time and space distribution of volcanic activity will lead to a modulation of the sputtering yields, should sputtering of volcanic plumes be important. Total plume area at the time of Voyager 1 encounter was only a few percent of the satellite surface area [Strom et al., 1979], so sputtering yields from plume material would have to be high in order to account for a significant fraction of the required source flux. On the dayside, assuming that the observation of $\mathrm{SO}_{2}$ gas reflects the presence of an atmosphere with a well-defined critical level, plume sputtering would be negligible if plume heights ( $\leqslant 300 \mathrm{~km}$ [Strom et al., 1979]) fall below the exobase, which could easily be the case. On the nightside, however, plume sputtering should occur. If most sputtering occurs at the top of the plume, then gaseous components will be removed in much the same way as from an atmosphere. Entrained particulates [Smith et al., 1979] will sputter essentially as if they were at rest on the surface. An enhancement of the sputtering yields will occur because of the decrease in gravitational acceleration $g$ with altitude, but for plume heights of a few hundred kilometers the effects are not large.

\section{SUMMARY}

The process of magnetospheric sputtering by corotating $S$ and $O$ ions seems capable of supplying a significant fraction of the particles from Io necessary to provide the source material for the observed $\mathrm{S}, \mathrm{O}, \mathrm{Na}$, and $\mathrm{K}$ clouds. Direct sputtering from an unprotected surface is marginally efficient enough to be a source of $S$ and $O$ ions, and this process may also be able to supply enough alkali atoms to maintain the $\mathrm{Na}$ and $\mathrm{K}$ clouds. Direct sputtering of a full-disk S-containing atmosphere with an exobase at a few hundred kilometers could provide up to $5 \times 10^{10} \mathrm{~S}$ atoms $\mathrm{cm}^{-2} \mathrm{~s}^{-1}$, which is compatible with estimates of the necessary emission flux. On a small satellite with a hot atmosphere, like Io, the exobase could extend as far as several Io radii, and under such conditions, $S$ and $O$ emission rates could be as large as $\sim 10^{12} \mathrm{~cm}^{-2} \mathrm{~s}^{-1}$.

If direct sputtering of the surface occurs, then any atomic species present in addition to $\mathrm{S}, \mathrm{O}, \mathrm{Na}$, and $\mathrm{K}$ must also be sputtered and should be present in the magnetosphere in the vicinity of Io. Sputtering also represents a potential source of large molecular species for the magnetosphere. Finally, the spatial and temporal variation of atomic and molecular emission fluxes may depend on a wide variety of conditions which at the present time we cannot adequately characterize. Local 
field effects can cause transport of both incident and emitted ions along nonballistic trajectories, and under special circumstances, enhanced cascade sputtering or the phenomenon of ionic saltation may occur. The inhomogeneity of the atmosphere can lead to large fluctuations in the sputtering rate, since surface and atmospheric yields can differ by up to several orders of magnitude. As a special case of this inhomogeneity, volcanic activity could modulate the emission rate, to the extent that the upper regions of the plumes are subject to sputtering.

Acknowledgments. One of us (P.K.H.) would like to acknowledge the interest and support of D. A. Bromley at Yale and T. A. Tombrello at Caltech. We also thank G. J. Wasserburg of Caltech's Lunatic Asylum for an intense discussion on the abundance of the elements. This work was supported in part by the U.S. Department of Energy (EY-76-C-02-3074) at Yale and the National Aeronautics and Space Administration (NGR 05-002-333), the Caltech President's Fund, and the National Science Foundation (PHY79-23638) at Caltech.

The Editor thanks D. Matson for his assistance in evaluating this paper.

\section{REFERENCES}

Benninghoven, A., Surface investigation of solids by the statical method of secondary ion mass spectroscopy (SIMS), Surf. Sci., 35, 427-457, 1973.

Biersack, J. P., and E. Santner, Sputtering of potassium chloride by $H$, He, and Ar ions, Nucl. Instrum. Methods, 132, 229-235, 1976.

Brown, R. A., Optical line emission from Io, Symp. Int. Astron. Union, 65, 527-531, 1974.

Brown, R. A., and Y. L. Yung, Io, its atmosphere and optical emissions, in Jupiter, edited by T. Gehrels, pp. 1102-1145, University of Arizona Press, Tucson, 1976.

Brown, W. L., L. J. Lanzerotti, J. M. Poate, and W. M. Augustyniak, 'Sputtering' of ice by MeV light ions, Phys. Rev. Lett., 40, 1027 1030, 1978.

Brown, W. L., W. M. Augustyniak, E. Brody, B. Cooper, L. J. Lanzerotti, A. Ramirez, R. Evatt, and R. E. Johnson, Energy dependence of the erosion of $\mathrm{H}_{2} \mathrm{O}$ ice films by $\mathrm{H}$ and $\mathrm{He}$ ions, Nucl. Instrum. Methods, 170, 321-325, 1980.

Carey, W. C., and J. A. M. McDonnell, Lunar surface sputter erosion: A Monte Carlo approach to microcrater erosion and sputter redeposition, Proc. Lunar Sci. Conf. 7th, 913-926, 1976.

Carlson, R. W., D. L. Matson, T. V. Johnson, and J. T. Bergstralh, Sodium D-line emission from Io: Comparison of observed and theoretical line profiles, Astrophys. J., 223, 1082-1086, 1978.

Chamberlain, J. N., Planetary coronae and atmospheric evaporation, Planet. Space Sci., 11, 901-960, 1963.

Fanale, F. P., R. H. Brown, D. P. Cruikshank, and R. N. Clake, Significance of absorption features in Io's IR reflectance spectrum, $\mathrm{Na}$ ture, 280, 761-763, 1979.

Griffith, J. E., R. A. Weller, L. E. Seiberling, and T. A. Tombrello, Sputtering of uranium tetrafiuoride in the electronic stopping region, Radiat. Eff., 51, 223-232, 1980.

Haff, P. K., Possible new sputtering mechanism in track registering materials, Appl. Phys. Lett., 29, 473-475, 1976.
Haff, P. K., and C. C. Watson, The erosion of planetary and satellite atmospheres by energetic atomic particles, J. Geophys. Res., 84, 8436-8442, 1979.

Haff, P. K., Z. E. Switkowski, and T. A. Tombrello, Solar wind sputtering of the Martian atmosphere, Nature, 272, 803-804, 1978.

Hapke, B., and W. Cassidy, Is the moon really as smooth as a billiard ball? Remarks concerning recent models of sputter-fractionation on the lunar surface, Geophys. Res. Lett., 5, 297-300, 1978.

Johnson, T. V., A. F. Cook II, C. Sagan, and L. A. Soderblom, Volcanic resurfacing rates and implications for volatiles on Io, Nature, 280, 746-750, 1979.

Johnson, T. V., G. Morfill, and E. Grün, Dust in Jupiter's magnetosphere: An Io source? paper presented at Colloquium 57, Int. Astron. Union, Kailua-Kona, Hawaii, May 1980.

Kumar, S., The stability of an $\mathrm{SO}_{2}$ atmosphere on Io, Nature, 280 , 758-760, 1979.

Kupo, I., Y. Mekler, and A. Eviatar, Detection of ionized sulfur in the Jovian magnetosphere, Astrophys. J., 205, L51-L53, 1976.

Matson, D. L., T. V. Johnson, and F. P. Fanale, Sodium D-line emission from Io: Sputtering and resonant scattering hypothesis, $A s-$ trophys. J., 192, L43-L46, 1974.

Matson, D. L., G. A. Ransford, and T. V. Johnson, Heat flow from Io (J1), J. Geophys. Res., 86, 1664-1672, 1981.

McElroy, M. B., and Y. L. Yung, The atmosphere and ionosphere of Io, Astrophys. J., 196, 227-250, 1975.

Pearl, J., R. Hanel, V. Kunde, W. Maguire, K. Fox, S. Gupta, C. Ponnamperuma, and $\mathrm{F}$. Raulin, Identification of gaseous $\mathrm{SO}_{2}$ and new upper limits for other gases on Io, Nature, 280, 755-758, 1979.

Pilcher, C. B., and J. S. Morgan, Detection of singly ionized oxygen around Jupiter, Science, 297-298, 1979.

Seiberling, L. E., J. E. Griffith, and T. A. Tombrello, Enhanced sputtering of dielectric materials and its relationship to track registration, Lunar Planet. Sci, XI, 1021-1023, 1980a.

Seiberling, L. E., J. E. Griffith, and T. A. Tombrello, A thermalized ion explosion model for high energy sputtering and track registration, Radiat. Eff., 52, 201-210, $1980 b$.

Sigmund, P., Theory of sputtering, I, Sputtering yield of amorphous and polycrystalline targets, Phys. Rev., 184, 383-416, 1969.

Smith, B. A., E. M. Shoemaker, S. W. Kieffer, and A. F. Cook II, The role of $\mathrm{SO}_{2}$ in volcanism on Io, Nature, 280, 738-743, 1979.

Smythe, W. D., R. M. Nelson, and D. B. Nash, Spectral evidence for $\mathrm{SO}_{2}$ frost or adsorbate on Io's surface, Nature, 280, 766, 1979.

Strom, R. G., R. J. Terrile, H. Masursky, and C. Hansen, Volcanic eruption plumes on Io, Nature, 280, 733-736, 1979.

Sullivan, J. D., and F. Bagenal, In situ identification of various ionic species in Jupiter's magnetosphere, Nature, 280, 798-799, 1979.

Trafton, L., Detection of a potassium cloud near Io, Nature, 258,690 692, $1975 a$.

Trafton, L., High resolution spectra of Io's sodium emission, Astrophys. J., 202, L107-L112, 1975 b.

Watson, C. C., P. K. Haff, and T. A. Tombrello, Solar wind sputtering effects on Mars and Venus, Lunar Planet. Sci., XI, 1022-1024, $1980 a$.

Watson, C. C., P. K. Haff, and T. A. Tombrello, Solar wind sputtering effects in the atmospheres of Mars and Venus, Proc. Lunar Planet. Sci. Conf. 11th, 2479-2502, $1980 b$.

(Received October 7, 1980; revised March 4, 1981; accepted March 5, 1981.) 